\title{
Research Ethics and the Future Direction of Sleep Medicine Research
}

\author{
Chol Shin, MD, PhD \\ Division of Pulmonary and Critical Care Medicine, Department of Internal Medicine, Korea University Ansan Hospital, Ansan, Korea
}

\author{
Correspondence \\ Chol Shin, MD, PhD \\ Division of Pulmonary and Critical Care \\ Medicine, Department of Internal Medicine, \\ Korea University Ansan Hospital, \\ 123 Jeokgeum-ro, Danwon-gu, \\ Ansan 15355, Korea \\ Tel +82-31-412-5603 \\ Fax +82-31-412-5604 \\ E-mail chol-shin@korea.ac.kr \\ ORCID \\ Chol Shin \\ https://orcid.org/0000-0002-2928-8576
}

It is important that researchers observe and maintain the research ethics for all of studies with regard to the safety of study participants and the competence of studies, as per the provisions of the Declaration of Helsinki. ${ }^{1}$ However, it should be noted that the standards apply not only to researchers, but also all journal editors or publishers by Committee on Publication Ethics. ${ }^{2}$ The Sleep Medicine Research, the official journal of Asian Society of Sleep Medicine and Korean Society of Sleep Medicine has made every effort to comply with the established global standards of research ethics. Furthermore, we reviewed all of 50 original clinical research articles, five animal research articles, sixteen case reports, and two image reports published in the Sleep Medicine Research about research ethics especially in terms of informed consent for the future direction of Sleep Medicine Research.

Among 50 original human research articles, 24 articles revealed that the authors gave informed consents to the participants, or waived by the Institutional Review Board (IRB). As for other 26 articles, 22 research studies, which were approved by the IRB but no mention on the informed consent in the manuscript, were done with retrospective medical chart review, previous cohort, national health insurance data and/or de-identified (redacted) hospital inpatient information. We've had contact with the authors of other four studies which were done prospectively and approved by IRB, but there was no comment on informed consent. The authors of two studies responded that informed consent was "waived by IRB," and the authors of other two studies answered that they "gave informed consent" to the participants but somehow neglected to mention this issue in the manuscript subsequently submitted. Of the five animal studies, the authors of four studies have revealed that the studies were "approved by the institutional committee." Weve had contact with the author of a single study, in which there was no comment on approval by the institutional committee. In response to inquiry, the author claimed that "the study was approved" but they somehow omitted that particular disclosure from the final report.

We also reviewed sixteen case reports and two image reports about the informed consent or verbal agreement of the subjects in the report. The author of one case report (in which individual's identifying information was openly disclosed in the article) answered that "the subjects agreed with the publication." However the authors of just five case reports in which subject's identifying information was not included in the reports answered that "the subjects agreed to the publication." The authors of other case reports admitted that they did not ask the subjects about the agreement with the publication, because identifiable information was not included in the manuscript. There is controversy as to whether the authors should or should not disclose, up front, awareness and agreement of subjects with the publication in the case reports. ${ }^{3}$ The International Committee of Medical Journal Editors ${ }^{4}$ requires informed consent of subjects for publication if subject's identifiable information is used, but does not address the issue as it relates to case reports. However, case reports sometimes include a rare disease, and subjects may be identifiable despite no mention of direct identifiable information. Since confidentiality is a bedrock obligation of researchers, reviewers and publishers, it seems very ap- 
propriate that we consider obtaining the informed consent from the subjects, not just of original studies but also of case reports.

In an ongoing effort to improve the publication ethics of Sleep Medicine Research, we changed publication process in several ways vis-à-vis this issue.

First, we ask the authors to disclose the acquisition of informed consent from participants, or waiver by IRB (not just for original research article but also for case reports). It is a decision for the future direction of Sleep Medicine Research. Second, ORCID ID will be asked for the researchers for credentials to identify the researchers' fitness. Third, author's contribution was asked to be revealed in the paper.

For the development and the bright future of Sleep Medicine Research, please review our privacy policy and give us gener- ous support.

\section{REFERENCES}

1. World Medical Association. Declaration of Helsinki: Medical research involving human subjects [cited 2017 Sep 24]. Available from: https:// www.wma.net/what-we-do/medical-ethics/declaration-of-helsinki/.

2. COPE. Codes of conduct and best practice guidelines [cited $2017 \mathrm{Sep}$ 24]. Available from: https://publicationethics.org/resources/code-conduct.

3. Gibson E. Publication of case reports: Is consent required? Paediatr Child Health 2008;13:666-7.

4. ICMJE. Uniform requirements for manuscripts submitted to biomedical journals: Writing and editing for biomedical publication - privacy and confidentiality [cited 2017 Sep 24]. Available from: http://www.icmje.org/recommendations/browse/roles-and-responsibilities/protection-of-research-participants.html. 